Collection SFN 9 (2008) 121-137

(C) EDP Sciences, Les Ulis

DOI: 10.1051/sfn:2008009

\title{
Ordre local
}

\author{
I. Mirebeau
}

Laboratoire Léon Brillouin, CEA-CNRS, CEA/Saclay, 91191 Gif-sur-Yvette, France

\begin{abstract}
Résumé. L'ordre local entre atomes ou moments magnétiques joue un rôle important en physique de la matiére condensée. La diffraction de neutrons constitue un outil de choix pour son étude microscopique. Ce chapître présente quelques cas simples, dans les solutions solides d'alliages binaires, d'ordres chimiques ou magnétiques locaux qui peuvent être facilement modélisés et fournir des informations quantifiables. Il présente aussi un bref aperçu de phénomènes dont l'ordre local est précurseur, comme la séparation de phase ou la transition vers un ordre à longue distance. Enfin il donne des exemples des composés géométriquement frustrés, où des ordres magnétiques locaux originaux, liquide de spin, glace de spin ou verre de spin chimiquement ordonné, sont stabilisés, et dans lequel l'état magnétique fondamental évolue sous pression.
\end{abstract}

\section{INTRODUCTION}

L'ordre local joue un rôle essentiel en physique de la matiére condensée, pour plusieurs raisons. D'une part, certaines propriétés physiques des matériaux comme la résistivité électrique ou thermique, la dureté ou la ductilité, sont liées à la structure locale. D'autre part, l'état d'ordre local constitue souvent un état précurseur d'une transition vers un ordre à longue portée. Ceci à telle enseigne que la stabilité thermodynamique d'une phases ordonnée (cristalline ou magnétique) est contrôlée par les potentiels d'interactions de paires entre atomes ou moments magnétiques proches voisins, qui ne peuvent être déterminés quem dans la solution solide ou dans la phase paramagnétique associée. Par convention, on appellera "local" un état d'ordre qui concerne des paires d'entités, - atomes, particules, moments magnétiques -, proches voisines. Du point de vue chimique (atomes, particules), ce chapitre concerne surtout l'ordre local d'occupations de sites dans un réseau cristallin, au détriment de l'ordre local topologique observé par exemple dans les liquides ou amorphes. Du point de vue magnétique, on s'intéressera aux corrélations de paires entre moments magnétiques proches voisins.

Notons tout de suite qu'ordre chimique local et ordre magnétique local ne sont pas forcément liés. Un alliage désordonné chimiquement peut présenter un état d'ordre magnétique à longue distance : c'est le cas d'un alliage ferromagnétique à un seul type d'atome magnétique par exemple. Dans un alliage binaire $\mathrm{AB}$ sur un réseau triangulaire, l'interaction $\mathrm{A}-\mathrm{B}$ hétérocoordinante (A préfère s'entourer de B) est frustrée du fait de la géométrie du réseau, ce qui peut favoriser le désordre chimique, mais pas nécessairement magnétique. Réciproquement, un composé parfaitement ordonné chimiquement, peut être incapable de s'ordonner magnétiquement à longue distance, par exemple sous l'influence de fluctuations quantiques ou lorsque les interactions magnétiques sont géométriquement frustrées. Une interaction de paires antiferromagnétique entre atomes premiers voisins identiques, situés sur un réseau triangulaire, est un exemple bien connu de frustration géométrique magnétique dans une structure chimiquement ordonnée.

Cette remarque mise à part, les modèles développés historiquement pour formaliser l'analyse de l'ordre magnétique et de l'ordre chimique local se sont souvent inspirés les uns des autres, la description de l'énergie du système à partir d'un Hamiltonien d'Ising étant formellement adaptée à traiter les deux cas. D'un point de vue expérimental, la diffraction des neutrons constitue un outil de choix pour l'étude de l'ordre local, le signal diffus, i.e. entre les pics de Bragg, étant directement relié à la transformée de Fourier des fonctions de corrélations de paires entre atomes ou moments magnétiques voisins. Des techniques classiques permettent en général de séparer les contributions chimiques et 
magnétiques à la section efficace. Elles tirent profit de l'influence des paramètres thermodynamiques $(\mathrm{P}, \mathrm{T}, \mathrm{H})$, ou jouent sur la géométrie du faisceau de neutrons par rapport à l'aimantation moyenne de l'échantillon, et éventuellement sur la polarisation des neutrons. Comme elles ne sont pas propres à l'étude de l'ordre local, elle sont simplement mentionnées briévement dans chaque cas.

Loin d'être exhaustif, ce chapitre se borne à décrire quelques exemples expérimentaux et modèles simples, permettant d'introduire la physique du comportement du matériau, et si possible de visualiser le type d'ordre local et d'intuiter comment l'analyser en l'absence de tout calcul. Dans les cas simples, comme les solution solides d'alliages binaires et/ou ferromagnétiques, il est possible de quantifier l'ordre local, par des paramètres d'ordre chimiques ou magnétiques, et d'avoir accés à des grandeurs intéressantes comme les potentiels de paires. Il est clair que c'est loin d'être toujours possible, et que la quantification de l'ordre local passe par des modèles souvent complexes. Des méthodes d'analyse non abordées ici, comme l'étude du fond diffus par des simulations "Monte Carlo Inverse" ou la modélisation de la section efficace par la fonction de corrélation de paires dérivée des liquides, sont maintenant trés utilisées pour analyser l'ordre local.

Le plan de ce chapitre est le suivant. Il commence par introduire les paramètres d'ordre chimique local et les potentiels d'interaction de paires, en considérant deux exemples, celui des alliages lacunaires titane-carbone et celui des alliages fer-chrome, et décrit briévement les mesures de cinétique de mise en ordre chimique local par résistivité électrique. Il présente ensuite quelques aspects de l'ordre magnétique local dans les alliages ferromagnétiques, systèmes sans désordre d'orientation des moments pour lesquels l'analyse de l'ordre magnétique peut être calquée sur celle de l'ordre chimique. Une brève incursion "du local vers le mésoscopique" permet d'introduire les aspects précurseurs de l'ordre local dans deux cas typiques : la séparation de phase et l'approche d'une transition critique. Enfin, des exemples récents de systèmes géométriquement frustrés, montrent des ordres magnétiques locaux originaux, liquides de spin, glaces de spin ou verres de spin sans désordre chimique, et leur transformation possible en ordres magnétiques à longue distance sous l'influence de la pression.

\section{ORDRE CHIMIQUE LOCAL}

L'arrangement local de deux ou plusieurs types d'atomes sur les sites d'un réseau cristallin constitue un problème bien connu de mécanique statistique. En diffraction de neutrons ou de rayons $\mathrm{X}$, un désordre d'occupation parfait (atomes disposés aléatoirement sur les sites du réseau) donne lieu à une intensité diffuse constante entre les pics de Bragg, appelée "incohérent de Laüe” Lorsqu'il existe un ordre local entre les différents types d'atomes, cette intensité est modulée dans l'espace réciproque.On ne considère ici que le cas trés simple où le réseau reste inchangé : il n'y a pas de déplacements atomiques liés à l'occupation des sites.

\subsection{Paramètres d'ordre chimique et potentiels de paires}

Les paramètres d'ordre local, qui permettent de décrire trés simplement la modulation du fond diffus, ont été introduits par Cowley [1] et Warren [12], en fonction des probabilités conditionnelles d'occupation. Dans le cas d'un alliage binaire $\mathrm{A}_{c_{A}} \mathrm{~B}_{c_{B}}$ en solution solide, les paramètres d'ordre $\alpha\left(R_{i}\right)$, notés plus simplement $\alpha_{i}$, sont définis par l'expression :

$$
\alpha_{i}=1-\frac{P_{A B}\left(R_{i}\right)}{c_{B}}=1-\frac{P_{B A}\left(R_{i}\right)}{c_{A}}
$$

où $\mathrm{P}_{A B}\left(\mathrm{R}_{i}\right)$ est la probabilité conditionnelle de trouver un atome $\mathrm{B}$ à une distance $\mathrm{R}_{i}$ d'un atome A supposé à l'origine et $c_{A}$ et $c_{B}$ les concentrations atomiques des espéces $\mathrm{A}$ et $\mathrm{B}\left(c_{A}+c_{B}=1\right)$. Les paramètres $\alpha$ décroissent quand la distance $\mathrm{R}$ augmente. Une tendance à la ségrégation de phase ou homocoordination (un atome "préfère" s'entourer d'atomes de même espèce) se traduit par des paramètres d'ordre entre proches voisins $\alpha_{i} \geq 0$. Une tendance à la formation de surtructure ordonnée 
ou hétérocoordination (un atome A "préfère" s'entourer d'atomes B et réciproquement) se traduit par $\alpha_{i} \leq 0$. Une répartition aléatoire des atomes $\mathrm{A}$ et $\mathrm{B}$ correspond à $\alpha_{i}=0$. Les paramètres d'ordre vérifient également les relations triviales $\alpha_{0}=1$ et $-c_{B} / c_{A} \leq \alpha_{i} \leq 1$ (pour $c_{B} \leq c_{A}$ ). La section efficace diffuse liée à l'ordre local est proportionnelle à la transformée de Fourier des paramètres d'ordre. Pour un monocristal, elle s'exprime comme :

$$
\frac{d \sigma}{d \Omega}=c_{A} c_{B}\left(b_{A}-b_{B}\right)^{2} \sum_{n} \alpha\left(\mathbf{R}_{\mathbf{n}}\right) \cdot \cos \left(\mathbf{Q} \cdot \mathbf{R}_{\mathbf{n}}\right)
$$

où $b_{A}$ et $b_{B}$ sont les longueurs de diffusion cohérentes des atomes $\mathrm{A}$ et $\mathrm{B}$ et où la somme s'étend sur les paires d'atomes séparées par un vecteur $\mathbf{R}_{\mathbf{n}}$. Dans un monocristal, l'intensité diffuse due à l'ordre local est donc périodique dans l'espace réciproque. Une tendance à l'homocoordination se traduit par des maxima diffus centrés sur l'origine du réseau réciproque et les pics de Bragg du réseau. Une tendance à l'hétérocoordination correspond à des maxima diffus en dehors des pics de Bragg du réseau, en général centrés sur les positions des pics de la structure ordonnée à longue distance sous-jacente. Dans un polycristal, la moyenne d'orientation du terme $\left\langle\cos \left(Q \cdot R_{i}\right)\right\rangle=\sin \left(Q \cdot R_{i}\right) / Q \cdot R_{i}$ conduit à une perte de périodicité de la section efficace, qui s'exprime alors comme :

$$
\frac{d \sigma}{d \Omega}=c_{A} c_{B}\left(b_{A}-b_{B}\right)^{2} \cdot\left[1+\sum_{i \neq 0} N_{i} \alpha\left(R_{i}\right) \cdot \frac{\sin \left(Q \cdot R_{i}\right)}{Q \cdot R_{i}}\right]
$$

où le premier terme correspond à la section efficace de Laüe liée à une répartition atomique aléatoire, et où la somme s'étend sur toutes les couches i situées à la distance $R_{i}$. Intuitivement, il est clair que les paramètres d'ordre dépendent de la température. L'agitation thermique aidant, la répartition atomique tend vers une répartition aléatoire quand la température augmente.

Le modèle de champ moyen développé par Clapp et Moss [3] permet de s'affranchir du facteur thermique pour obtenir à partir des paramètres d'ordre les grandeurs caractéristiques du système, à savoir les potentiels de paires effectifs $V_{i j}=\frac{1}{2}\left(V_{i j}^{A A}+V_{i j}^{B B}-2 V_{i j}^{A B}\right)$ en fonction de la paire d'atomes considérée. Pour une paire donnée (A, B) en sites (i, j), $V_{i j}^{A B}$ est l'énergie d'interaction entre l'atome A et l'atome B sur les sites i et $\mathbf{j}$ séparés par le vecteur $\mathbf{R}_{\mathbf{n}}$. Cette énergie est supposée symétrique par rapport à l'échange des sites. Avec cette définition, couramment utilisée en chimie des alliages, si $\mathrm{V}_{i j}$ est négatif, le système "préfère" des atomes de même type sur les sites i et $\mathrm{j}$, et si $\mathrm{V}_{i j}$ est positif des atomes de types différents.

L'énergie de configuration du système s'exprime simplement à partir du potentiel effectif et des coefficients d'occupation atomiques par un Hamiltonien d'Ising [3]. Celui-ci s'écrit sous la forme :

$$
H=\frac{1}{4} \sum_{i, j} V_{i j} \cdot \bar{\sigma}_{i} \cdot \bar{\sigma}_{j}
$$

où les opérateurs $\bar{\sigma}$ sont des coefficients d'occupation, dont la moyenne thermodynamique est reliée aux concentrations atomiques et aux paramêtres d'ordre local [3]. L'expression de l'énergie ci-dessus est donc semblable à celle d'un système magnétique, les potentiels de paires effectifs jouant le rôle des interactions d'échange, et les opérateurs d'ocupation le rôle des spins. Elle montre bien la similitude formelle entre homocoordination et ferromagnétisme d'une part, hétérocoordination et antiferromagnétisme, d'autre part. La minimisation de l'énergie libre en champ moyen conduit à la relation :

$$
\alpha(\mathbf{Q}, T)=\frac{C}{1+\frac{2 \cdot c_{A} c_{B} V(\mathbf{Q})}{k T}}
$$

où $\mathrm{C}$ est une constante, $\mathrm{V}(\mathbf{Q})$ la transformée de Fourier du potentiel de paires et $\alpha(\mathbf{Q})$ la section efficace liée à l'ordre local, normalisée à la valeur de Laüe $c_{A} c_{B}\left(b_{A}-b_{B}\right)^{2}$ qui correspond à une répartition aléatoire des atomes A et B sur les sites du réseau. L'approximation de champ moyen est d'autant 


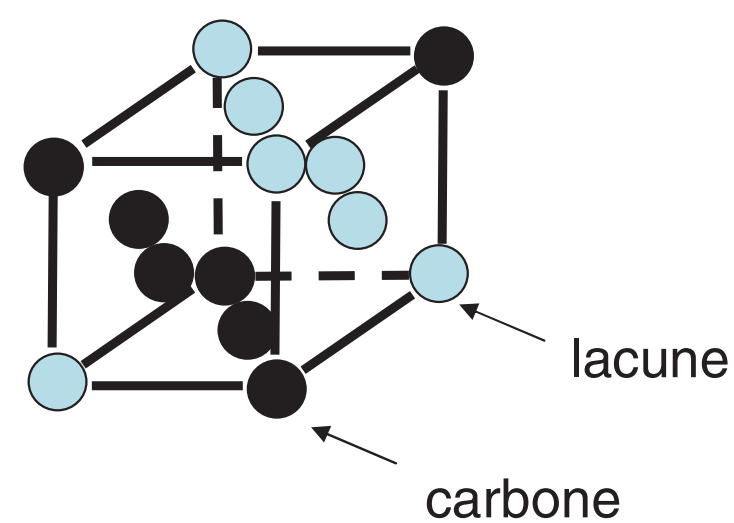

Figure 1. Ordre à longue distance des lacunes à la concentration stoechiométrique $\mathrm{TiC}_{0.5}$, montrant l'alternance de plans lacunaires et de plans de carbone perpendiculairement à un axe de type $\langle 111\rangle$.

plus valide que la température de mesure est élevée, par rapport à la température de transition vers la surstructure ordonnée ou la ségrégation de phase, si elle existe. Les potentiels de paires effectifs peuvent aussi être déduits des paramètres d'ordre par des méthodes statistiques standard, comme la méthode de variation d'amas ou les simulations Monte-Carlo [4]. Ils peuvent être comparés à ceux déduits de calculs de structure électronique. Ils jouent un rôle prépondérant dans la compréhension de la stabilité thermodynamique des phases cristallines et le calcul des diagrammes de phase. Les exemples suivants illustrent briévement quelques aspects expérimentaux reliés à cette problématique.

\subsection{Ordre de lacunes dans un monocristal de titane-carbone}

Nombre de carbures et nitrures de métaux de transition prèsentent une structure de type $\mathrm{Na}-\mathrm{Cl}: 2$ sousréseaux cubiques à faces centrées décalés d'un vecteur [ $1 / 200$. Mais tandis que le sous-réseau métal est en général parfaitement occupé, le sous-réseau métalloïde peut présenter un nombre important de lacunes, jusque vers 50\%. Pour certaines compositions, on peut obtenir des phases ordonnées. Autour de la composition stoechiométrique $\mathrm{Ti}_{2} \mathrm{C}$ les lacunes peuvent former une surstructure ordonnée à longue distance de vecteur de propagation $\mathrm{k}=(1 / 2,1 / 2,1 / 2)$, constituée par l'alternance de plans $\left(\begin{array}{lll}1 & 1 & 1\end{array}\right)$ vides et occupés (Fig. 1). A haute température, ou en s'éloignant de la composition stoechiométrique, on observe une solution solide entre lacunes et carbones, qui présente un ordre local marqué, avec des maxima d'intensité diffuse aux positions (1/2 1/2 1/2) et équivalentes du réseau réciproque (Fig. 2).

Il faut noter que les lacunes induisent aussi des déplacements atomiques : les premiers voisins métalliques s'éloignent des lacunes tandis que les seconds s'en approchent. C'est la raison de l'écart à la périodicité observé sur la Fig. 2 (haut). Le formalisme des déplacements statiques locaux [6] permet de déterminer et donc d'éliminer la contribution des distorsions locales du réseau, et d'avoir accés à celle de l'ordre local (Fig. 2 milieu). Dans le cas de telles mesures sur monocristaux à l'équilibre thermodynamique, un ajustement de la section efficace par l'équation (1) montre qu'une vingtaine de paramètres d'ordre est nécessaire pour ajuster correctement l'intensité expérimentale.

En revanche la détermination des potentiels de paires effectifs montre que quatre potentiels seulement, ceux associés aux quatre premières distances, suffisent à reproduire la section efficace mesurée [7, 8]. Les potentiels de paires peuvent être déterminés en champ moyen de point (équation 3), qui donne de très bons rapports entre les différents potentiels (et donc accès à la stabilité relative des phases ordonnées). Mais ils sont généralement obtenus par des méthodes plus sophistiquées de mécanique statistique (Monte Carlo Inverse, champ moyen d'amas) qui donnent de meilleures valeurs absolues et reproduisent donc mieux les températures de transition. Dans cet exemple les potentiels déterminés sont en bon accord avec ceux prédit par des calculs de structure électronique ab-initio. 

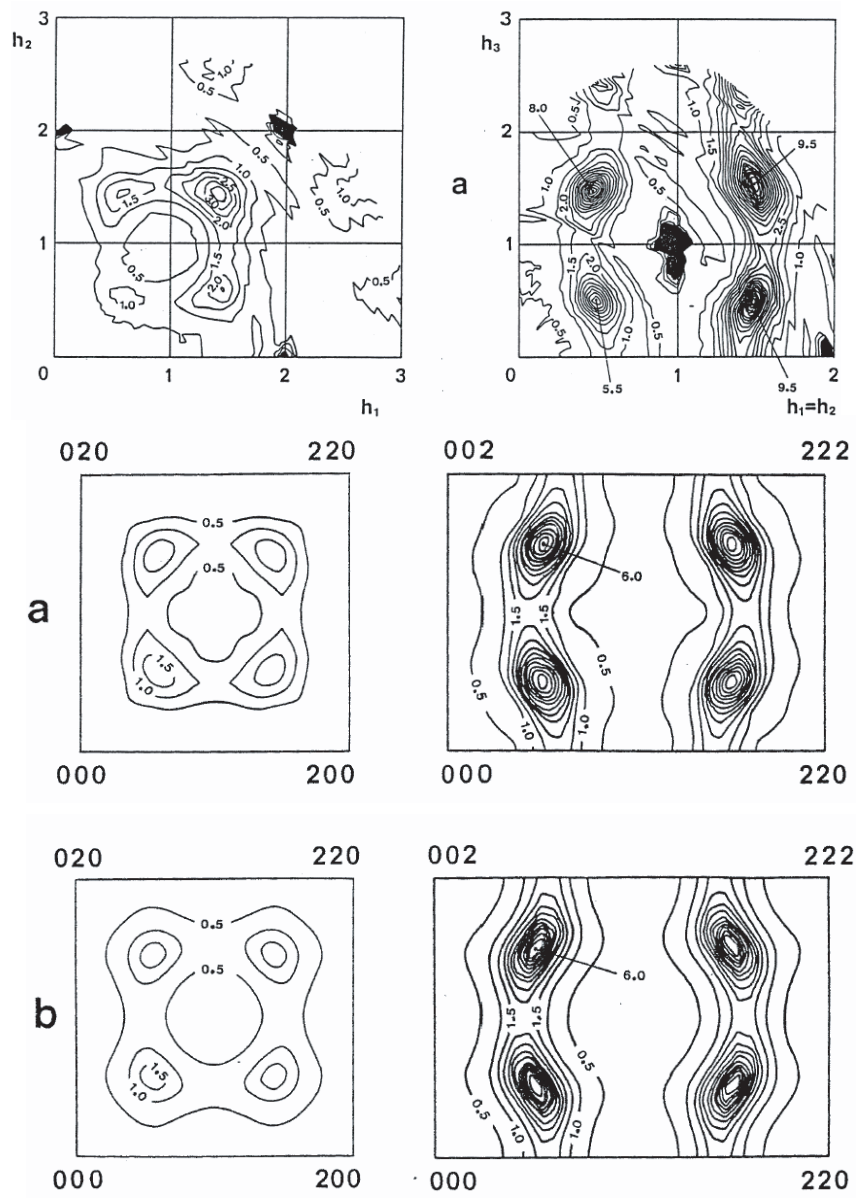

Figure 2. Cartes iso-intensité de la diffusion diffuse dans l'échantillon $\mathrm{TiC}_{0.64}$ à $900^{\circ} \mathrm{C}$ montrant la présence de maxima diffus en positions $(1 / 21 / 21 / 2)$ et équivalentes du réseau réciproque dans un alliage $\mathrm{TiC}_{0.64}$. À gauche : plan $\left(\begin{array}{lll}0 & 0 & 1\end{array}\right)$, à droite plan $(1-10)$. En haut l'intensité expérimentale, comprenant les contributions d'ordre et de distorsions locales du réseau. Au milieu : la contribution l'ordre local, périodique dans l'espace réciproque, calculée à partir d'une vingtaine de paramètres d'ordre ; en bas, intensité calculée à partir de quatre potentiels de paires effectifs $\left(\mathrm{V}_{1}=15 \mathrm{meV}, \mathrm{V}_{2}=48 \mathrm{meV}, \mathrm{V}_{3}=-4 \mathrm{meV}, \mathrm{V}_{4}=8 \mathrm{meV}\right.$. Résultats de la référence [7]).

\section{3 Étude de la cinétique d'ordre local par résistivité électrique}

Il n'est pas toujours possible, comme dans l'exemple précèdent, de mesurer la section efficace d'ordre local à l'équilibre thermodynamique. Ceci implique en général des mesures à haute température (plusieurs centaines de degrés Celsius), qui peuvent être incompatibles avec l'application d'un champ magnétique, permettant d'isoler la contribution magnétique. Les mesures à haute température nécessitent aussi l'utilisation de techniques "temps de vol" pour éliminer la contribution importante des phonons. L'alternative consiste à mesurer l'ordre local sur des alliages trempés, ce qui suppose une bonne connaissance préalable de la cinétique de mise en ordre. Les mesures de résistivité électrique $[9,10]$. permettent de déterminer cette cinétique, qui suit une loi d'activation thermique. Elles tirent parti du fait que la résistivité électrique résiduelle (mesurée à $\mathrm{T} \sim 0$ ) est déterminée par les paramètres d'ordre local (elle varie en gros comme $\alpha_{1}$ ) [11]. 


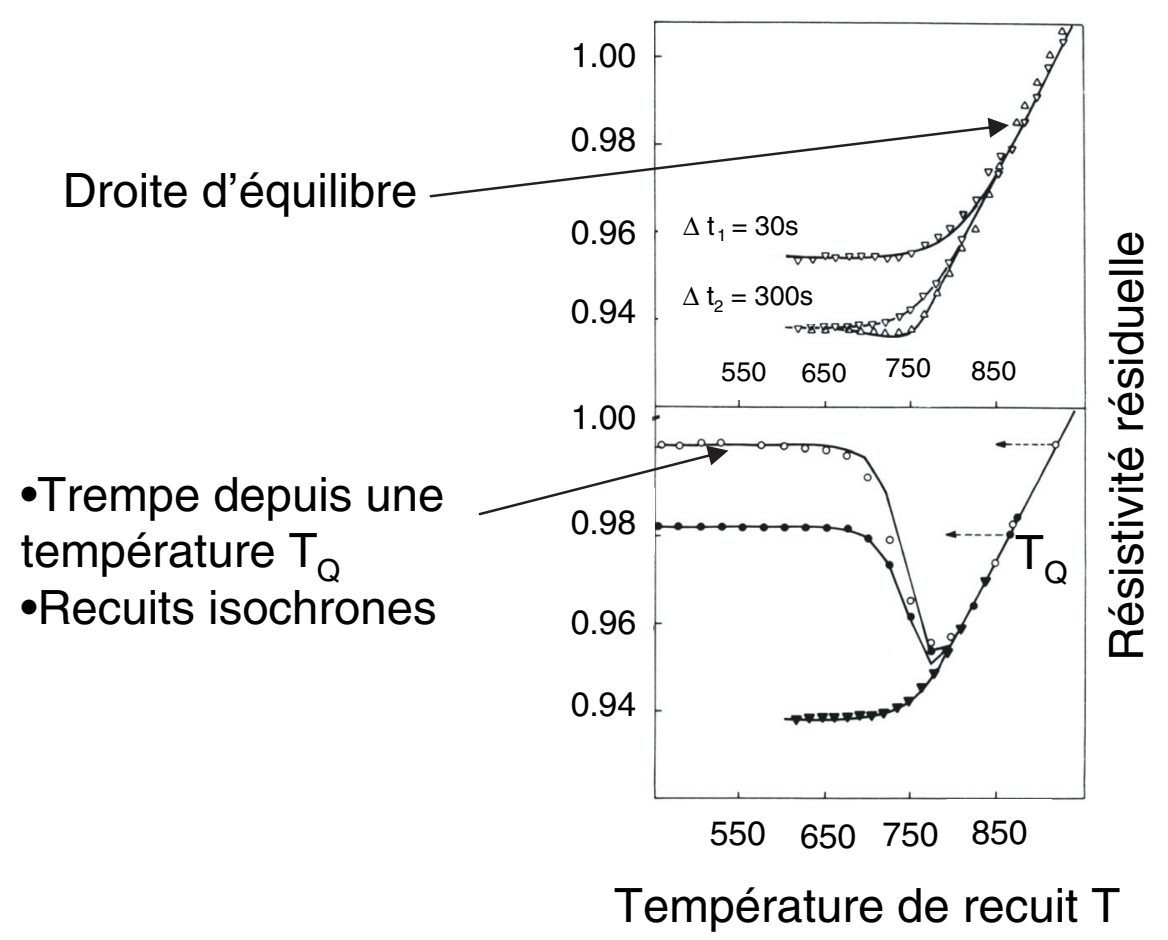

Figure 3. Cinétique d'ordre local dans un alliage $\mathrm{Fe}_{0.9} \mathrm{~V}_{0.1}$ : variation de la résistance à $4 \mathrm{~K}$ (relative à sa valeur aprés une trempe à $920 \mathrm{~K}$ ), en fonction de la température de recuit lors de recuits isochrones. En haut, la résistance décroit linéairement dans le domaine de température où l'échantillon est à l'équilibre thermodynamique, et présente un palier lorsque celui-ci n'est plus atteint pendant le temps de recuit $\Delta \mathrm{t}$ considéré $\left(\Delta \mathrm{t}_{1}=30 \mathrm{~s}, \Delta \mathrm{t}_{2}=300 \mathrm{~s}\right)$. Les cyles de température croissantes (triangle vers le haut) et décroissantes (triangle vers le bas) montrent une hystérèse à l'approche du palier. En bas : évolution de la résistance lors d'une trempe suivie de recuits. La trempe est effectuée depuis une température $\mathrm{T}_{\mathrm{Q}}$ de $920 \mathrm{~K}$ (ronds blancs) ou $872 \mathrm{~K}$ (ronds noirs). Les recuits ultérieurs à la trempe, de durée $\Delta \mathrm{t}_{2}=300 \mathrm{~s}$, permettent à la résistance de recouvrer sa valeur d'équilibre. Les lignes continues correspondent aux courbes calculées avec une énergie d'auto-diffusion $\mathrm{E}=2.66 \mathrm{eV}$. D'après la référence [10].

La méthode consiste à chauffer l'échantillon, laminé en ruban d'une dizaine de microns d'épaisseur, en faisant passer un courant électrique à une température de recuit donnée (contrôlée par l'intensité du courant) pendant un temps donné. L'échantillon est situé prés d'une surface d'hélium liquide, dans les vapeurs d'hélium, ce qui permet d'effectuer une trempe ultra rapide (plusieurs milliers de degrés/seconde) par coupure de courant. La résistance résiduelle est ensuite mesurée à $4 \mathrm{~K}$. La mesure de résistance résiduelle lors d'une succession de recuits isochrones à différentes températures (Fig. 3) permet de déterminer l'énergie d'activation de la cinétique de mise en ordre, et donc de choisir les conditions optimales (température, temps de recuit et méthode de trempe) afin d'obtenir et conserver l'état d'équilibre à une température bien définie. On peut remarquer que compte tenu des vitesses de trempe typiques des échantillons massifs (quelques dizaines de degré/seconde) et des énergies d'activation (quelques eV), la plage de température permettant de tremper un état d'ordre local avec un temps de recuit raisonable est assez étroite (une cinquantaine de degrés typiquement).

\subsection{Inversion de l'ordre local dans les alliages de fer-chrome}

Les solutions solides de fer-chrome, dans la phase cubique centrée riche en fer, présentent un cas original d'inversion de la nature de l'ordre local en fonction de la concentration [12]. A faible concentration de 

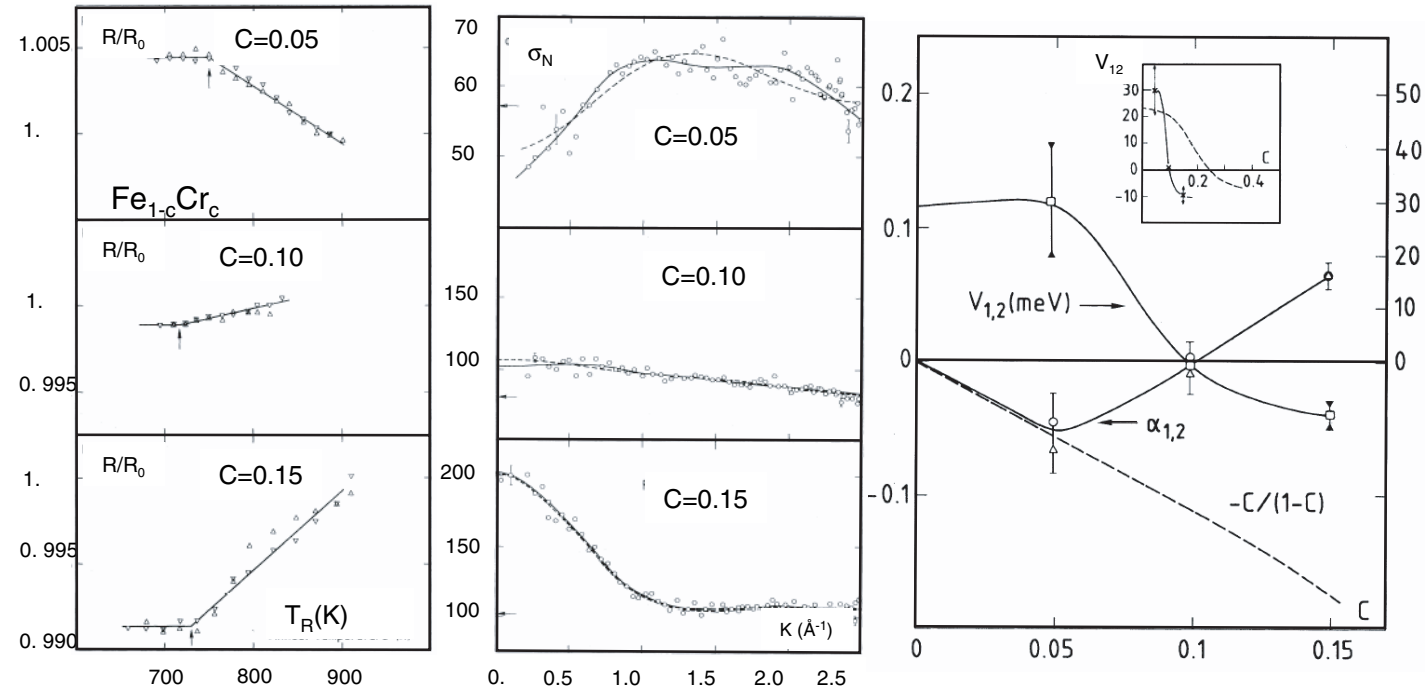

Figure 4. À gauche : cinétique d'ordre local dans les alliages $\mathrm{Fe}_{1-c} \mathrm{Cr}_{c}$ : variation de la résistance à $4 \mathrm{~K}$ (relative à sa valeur après une trempe depuis la température la plus haute) en fonction de la température de recuit. Au centre, section efficace de diffusion diffuse nucléaire dans les alliages $\mathrm{Fe}_{1-c} \mathrm{Cr}_{c}$ en fonction du vecteur de diffusion $\mathrm{K}(4 \pi \sin \theta / \lambda)$. Les flèches indiquent la valeur calculée de la section efficace incohérente (nucléaire + Laue). Les lignes sont des ajustements à 3(--) ou quatre (-) paramètres d'ordre $\alpha$. L'inversion de la nature de l'ordre local est visible sur la résistance (par le changement de signe de la pente de la droite d'équilibre) et sur l'intensité diffusée par les neutrons (par l'allure de la courbe aux petites valeurs de K). À droite, variation du paramètre d'ordre moyenné sur les deux premières couches de voisins en fonction de la concentration $\mathrm{c}$, et du potentiel de paires $\mathrm{V}_{1,2}(\mathrm{meV})$. La courbe $-c /(1-c)$ indique la limite de répulsion totale entre atomes de même espèce. Inset : Comparaison entre le potentiel de paires experimental (- -) et celui calculé (-) dans la référence [13]. D'après la référence [12].

chrome (c $<0.1)$, les atomes de chrome "se repoussent" $(\alpha<0)$, alors qu'à plus forte concentration (c $>0.1$ ), ils "s'attirent" $(\alpha>0)$. Cet effet surprenant a été prédit avant observation par un calcul de structure de bande [13]. Il est clairement visible sur les mesures de cinétique d'ordre local par résistance électrique : le changement de signe du paramètre d'ordre $\alpha_{1,2}$ moyenné sur les deux premières couches de voisins induit un changement de signe de la pente de la droite d'équilibre thermodynamique (Fig. 4). En diffusion de neutrons, la section efficace liée à l'ordre local, mesurée sur des alliages trempés depuis $703 \mathrm{~K}$, décroît aux petits valeurs du vecteur de diffusion $\mathrm{Q}$, pour la concentrations $\mathrm{c}=0.05$ : il $\mathrm{y}$ a tendance à la formation d'une surstructure ordonnée. Pour $\mathrm{c}=0.15$, l'augmentation de la section efficace à petits $\mathrm{Q}$ revèle une tendance à la segrègation.

Pour c $\sim 0.1$, l'intensité due à l'ordre local est voisine de celle calculée en supposant une distribution aléatoire. Les alliages $\mathrm{Fe}-\mathrm{Cr}$ de concentrations voisines de la concentration d'inversion présentent des applications potentielles pour les cuves des nouveaux réacteurs nucléaires ainsi que pour la fusion (projet ITER), car ils sont susceptibles de résister à de hautes températures et de fortes irradiations sans ségréger. L'étude de l'ordre local dans Fe-Cr suscite donc actuellement de nombreux travaux [14, 15].

\section{ORDRE MAGNÉTIQUE LOCAL DANS LES ALLIAGES FERROMAGNÉTIQUES}

Dans les alliages ferromagnétiques colinéaires, les inhomogénéités spatiales de la distribution d'aimantation donnent lieu à une diffusion diffuse magnétique analogue à la diffusion nucléaire liée à l'ordre chimique : on considére ici un modèle à une composante de spin. Le modèle le plus simple pour 
un alliage binaire $\mathrm{A}_{c_{A}} \mathrm{~B}_{c_{B}}$ (modèle de Shull-Wilkinson [16]) consiste à supposer qu'il n'y a que deux types de moments $\mathrm{m}_{A}$ et $\mathrm{m}_{B}$ répartis au hasard : la section efficace de diffusion magnétique s'exprime alors comme :

$$
\frac{d \sigma}{d \Omega}=c_{A} c_{B}\left(\frac{\gamma r_{0}^{2}}{2}\right)^{2}\left(1-(\mathbf{q} \cdot \mathbf{n})^{2}\right)\left(m_{A} f_{A}(Q)-m_{B} f_{B}(Q)\right)^{2}
$$

Cette expression est analogue à celle de la section efficace de Laüe pour une répartition atomique aléatoire, les moments magnétiques locaux jouant ici le rôle des longueurs de diffusion, à ceci prés qu'elle prend aussi en compte le facteur de forme magnétique $\mathrm{f}(\mathrm{Q})$ des atomes $\mathrm{A}$ et $\mathrm{B}$, (les distributions électroniques qui induisent les moments ne sont pas ponctuelles) et l'anisotropie intrinséque de l'interaction magnétique (seules les composantes des moments perpendiculaires au vecteur de diffusion $\mathbf{Q}$ sont impliquées dans la section efficace). Cette anisotropie est prise en compte par le terme d'orientation $1-(\mathbf{q} \cdot \mathbf{n})^{2}$ où $\mathbf{q}$ et $\mathbf{n}$ sont des vecteurs unitaires dans la direction du vecteur de diffusion et des moments magnétiques respectivement. Le terme d'orientation prend la valeur $2 / 3$ quand les domaines ferromagnétiques sont orientés au hasard.

En fait dans la plupart des cas, ce modèle trés simple ne permet pas de rendre compte de la réalité, et on doit introduire des paramètres d'ordre magnétiques. Si les moments sont colinéaires (alliages ferromagnétiques ou alignés par un champ), les paramètres d'ordre magnétiques $\phi_{i}$, dits de Marshall [17], correspondent à une variation de la "longueur" des moments dans l'espace.

La section efficace de diffusion diffuse magnétique est isolée de son équivalent chimique en utilisant plusieurs orientations du champ magnétique (qui aligne les domaines magnétiques), par rapport au vecteur de diffusion $\mathbf{Q}$ (typiquement $\mathbf{H}=0, \mathbf{H} / / \mathbf{Q}$ et $\mathbf{H} \perp \mathbf{Q}$ où le facteur d'orientation prend respectivement les valeurs $2 / 3,0$ et 1 ).

Sa mesure permet de déterminer dans l'alliage : i) les valeurs algébriques des moments magnétiques moyens $\mathrm{m}_{A}$ et $\mathrm{m}_{B}$ : on mesure en fait le contraste $\mathrm{m}_{A}-\mathrm{m}_{B}$, dont on peut obtenir le signe en utilisant des neutrons polarisés, et on obtient les moments ndividuels en combinant cette mesure à celle de l'aimantation moyenne $\mathrm{c}_{A} \mathrm{~m}_{A}+\mathrm{c}_{B} \mathrm{~m}_{B}$; ii) les perturbations sur ces moments liées à l'environnement local. Si l'alliage est dilué $(\mathrm{c} \ll 1)$, on peut négliger les perturbations sur le soluté par rapport à celles sur la matrice. La fonction de distribution des moments s'exprime alors simplement comme $\mathrm{M}(\mathrm{Q})=\mathrm{m}_{A}-\mathrm{m}_{B}+\sum_{i} \phi_{i} \sin \left(\mathrm{QR}_{i}\right) /\left(\mathrm{QR}_{i}\right)$ où $\phi_{i}$ est la perturbation du moment de la matrice causée par le soluté à la distance $R_{i}$. Ce type d'expérience a permis de déterminer les moments locaux dans les solutions solides à base de nickel et de fer [18, 19]. Moments locaux et perturbations magnétiques peuvent être comparés avec des calculs de structure de bande [20]. Dans les solutions solides diluées de fer-chrome (Fig. 5) on peut ainsi observer que le chrome possède un moment induit d'environ $0.8 \mu_{B}$ antiparallèle au moment de la matrice de fer, d'environ $2.2 \mu_{B}$, et perturbe localement les moments des couches d'atomes de fer voisins $[19,21]$. Il diminue les moment de fers premiers et seconds voisins $\left(\phi_{12}<0\right)$ et augmente celui des fers troisièmes voisins $\left(\phi_{3}>0\right)$. De façon générale, les perturbations sont plus fortes et plus localisées dans les alliages de fer que dans ceux de nickel.

Lorsque les moments corrélés à courte portée ne sont pas colinéaires, la section efficace diffuse liée à l'ordre magnétique local peut être modèlisée par des paramêtres d'ordre magnétiques qui font intervenir les corrélations de spins proches voisins, et donc les angles entre ces moments. De tels modèles ont été développés pour les verres de spins [22, 23]. Pour un polycristal, la section efficace magnétique, à laquelle on a soustrait sa valeur en région paramagnétique, qui correspond à la diffusion par des moments isolés, s'exprime comme précèdemment par une somme de fonctions $\left(\sin \left(\mathrm{QR}_{i}\right)\right) /\left(\mathrm{QR}_{i}\right)$, pondérées par des combinaisons linéaires des paramètres d'ordre magnétiques. Son ajustement permet de remonter aux corrélations de spin et dans les cas favorables, d'évaluer les interactions d'échange entre moments proches voisins. Un exemple détaillé est donné en ref. [23]. 

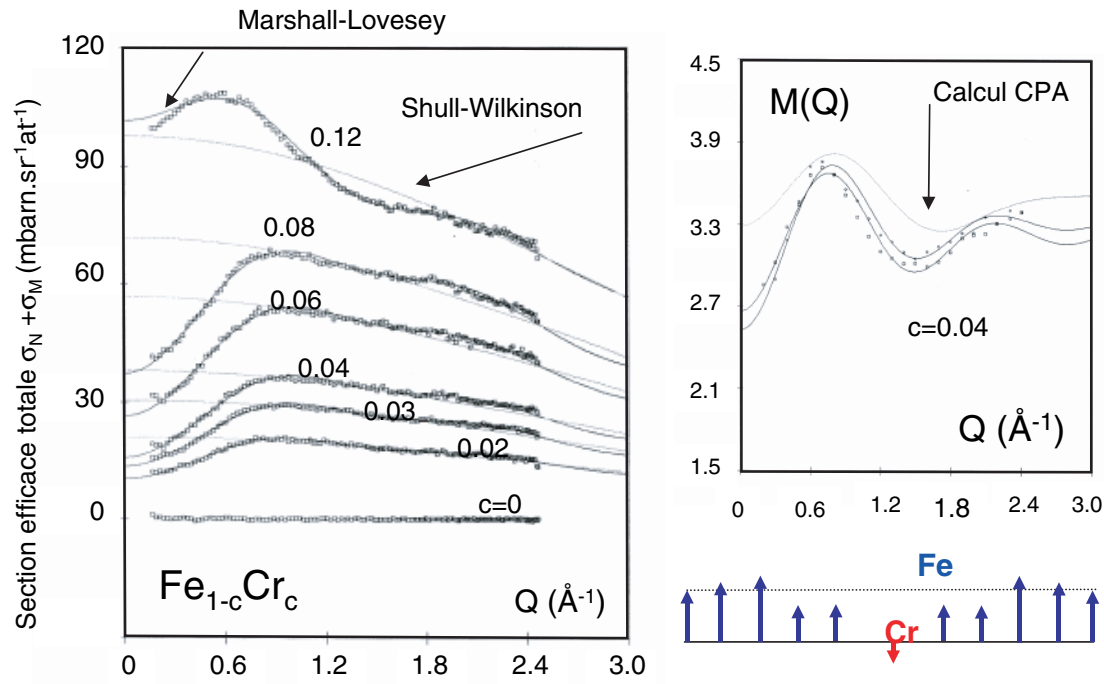

Figure 5. À gauche : section efficace de diffusion diffuse totale (nucléaire + magnétique) dans des alliages de fer-chrome en fonction du vecteur de diffusion Q. Ajustements par le modèle de Schull-Wilkinson (la dépendance en $\mathrm{Q}$ vient seulement du facteur de forme magnétique), et par le modèle de Marshall faisant intervenir les paramètres d'ordre chimique et magnétiques. A droite fonction de distribution locale des moments magnétiques $\mathrm{M}(\mathrm{Q})$ déduite de ces mesures : sa valeur moyenne est égale au contraste $\mathrm{m}_{\mathrm{Fe}}-\mathrm{m}_{\mathrm{Cr}}$, les modulations font intervenir les paramètres d'ordre magnétiques. Les courbes sont des ajustements par les paramètres d'ordre magnétiques et le résultat d'un calcul ab-initio [20] dans l'approximation du potentiel cohérent (CPA). En bas, schéma de principe de la distribution locale des moments sur les premières couches de d'atomes de fer voisins d'un moment de chrome. D'aprés [21].

\section{DU LOCAL AU MÉSOSCOPIQUE}

L'ordre local est souvent précurseur d'une transition de phase, et son étude permet parfois d'intuiter la nature de cette transition. D'un point de vue chimique, l'homocoordination sur les premiers voisins préjuge d'une ségrégation de phase, alors que l'hétérocoordination peut être le signe précurseur d'une transition vers une surstructure. D'un point de vue magnétique, les tendances au ferromagnétisme (resp. à l'antiferromagnétisme) observées en phase paramagnétique dans les corrélations de spin à courte portée, précèdent l'apparition à plus basse température des ordres à longue portée associés.

Les deux exemples ci-dessous permettent de visualiser ces phénomènes dans des cas concrets. Comme la taille des inhomogénéités concernées est de l'ordre de 10 à $100 \AA$ Å, celles-ci peuvent être observées par diffusion de neutrons aux petits angles, près de l'origine du réseau réciproque [24].

\subsection{Ordre local de particules}

Dans les alliages concentrés de fer-chrome $(c>0.10)$ qui présentent en solution solide une tendance à l'homocoordination, une trempe depuis la solution solide suivie d'un recuit induit la formation de précipités (Fig. 6) riches en chrome dans une matrice appauvrie en chrome : il y a séparation de phase. La cinétique de croissance de ces précipités est thermiquement activée, puisqu'elle implique une migration atomique, et donc régie comme dans la solution solide par la température et le temps de recuit. Si la densité des amas est suffisamment élevée, on voit apparaître un nouveau type d'ordre local, impliquant cette fois-ci les amas et non plus les atomes : la croissance d'un précipité riche en chrome induit au voisinage de celui-ci une région de déplétion où la concentration en chrome est plus faible que la concentration moyenne, d'où la présence d'une distance minimale d'approche $\mathrm{d}_{\text {min }}$ lors de la formation des amas (Fig. 6 à gauche). 

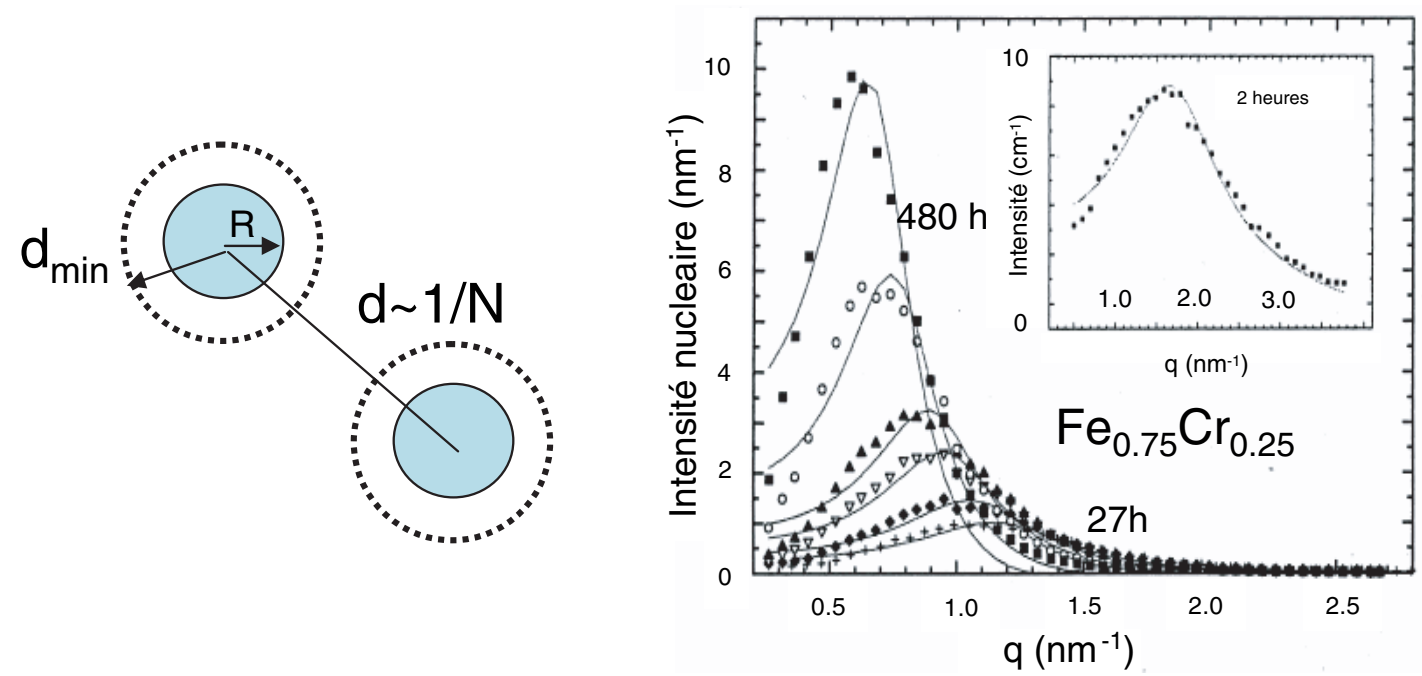

Figure 6. À gauche : ordre local de particules, montrant les trois distances caractéristiques de l'ordre local entre particules : $\mathrm{R}$ rayon de la particule, $\mathrm{d}_{\min }$ distance minimale d'approche, et d distance moyenne entre particules. À droite : intensité due à la diffusion nucléaire dans les alliages de fer-chrome $\mathrm{Fe}_{0.75} \mathrm{Cr}_{0.25}$ ségrégés, en fonction du vecteur de diffusion, pour différents temps de recuit. La position du pic d'interférence est surtout déterminée par $\mathrm{d}_{\text {min }}$. D'après [25].

L'intensité des neutrons diffusés est analogue à celle d'un liquide de sphéres dures. Autrement dit, c'est le produit du facteur de forme d'un amas $F(R)$ où $R$ est le rayon moyen de l'amas, d'une fonction d'interférence $\mathrm{I}\left(\mathrm{d}, \mathrm{d}_{\min }\right)$ où $\mathrm{d}$ est la distance moyenne entre amas (qui varie comme l'inverse du nombre d'amas $\mathrm{N}$ ), et où $\mathrm{d}_{\min }$ joue le rôle de la distance entre premiers voisins dans un liquide, et d'un terme de contraste. Ce dernier terme est proportionnel au carré des différences de densité de longueur de diffusion entre les deux milieux.

Dans les solutions solides de fer-chrome en phase ségrégée, la fonction d'interférence présente un pic vers $0.1 \AA^{-1}$, visible en diffusion aux petits angles. Lorsque le temps de recuit augmente, les précipités grossissent et la position du pic d'interférence (qui varie en gros comme $1 / \mathrm{d}_{\min }$ ) se déplace vers les petits vecteurs de diffusion.

Parallèlement au contraste nucléaire lié à la diffusion par les noyaux, il peut aussi exister un contraste magnétique entre les deux milieux. Pour que cela donne lieu à un pic d'interférence magnétique, il faut qu'il y ait cohérence entre les contributions magnétiques des précipités. C'est le cas dans les précipités de fer-chrome sous champ magnétique saturant, et la diffusion magnétique est alors le "miroir" de la diffusion chimique : elle montre, au facteur de forme près, la même dépendance avec le vecteur de diffusion [25]. Elle peut être isolée de celle-ci en jouant sur l'orientation du champ magnétique, comme au paragraphe précèdent. En revanche, si les précipités sont paramagnétiques ou superparamagnétiques, et non orientés mutuellement dans une matrice non magnétique, le pic d'interférence n'est vu que sur la contribution chimique. Dans ce cas, il peut être utile de coupler rayons X et neutrons pour séparer les contributions chimiques et magnétiques à l'intensité diffusée. Ceci a été fait par exemple pour des précipités de fer dans une matrice d'alumine [27].

\subsection{Approche d'un phénomène critique}

Dans les alliages de palladium-fer et palladium cobalt, la matrice de palladium présente une forte susceptibilité magnétique. Un atome Fe ou Co induit sur les Pd voisins un nuage de polarisation ou 

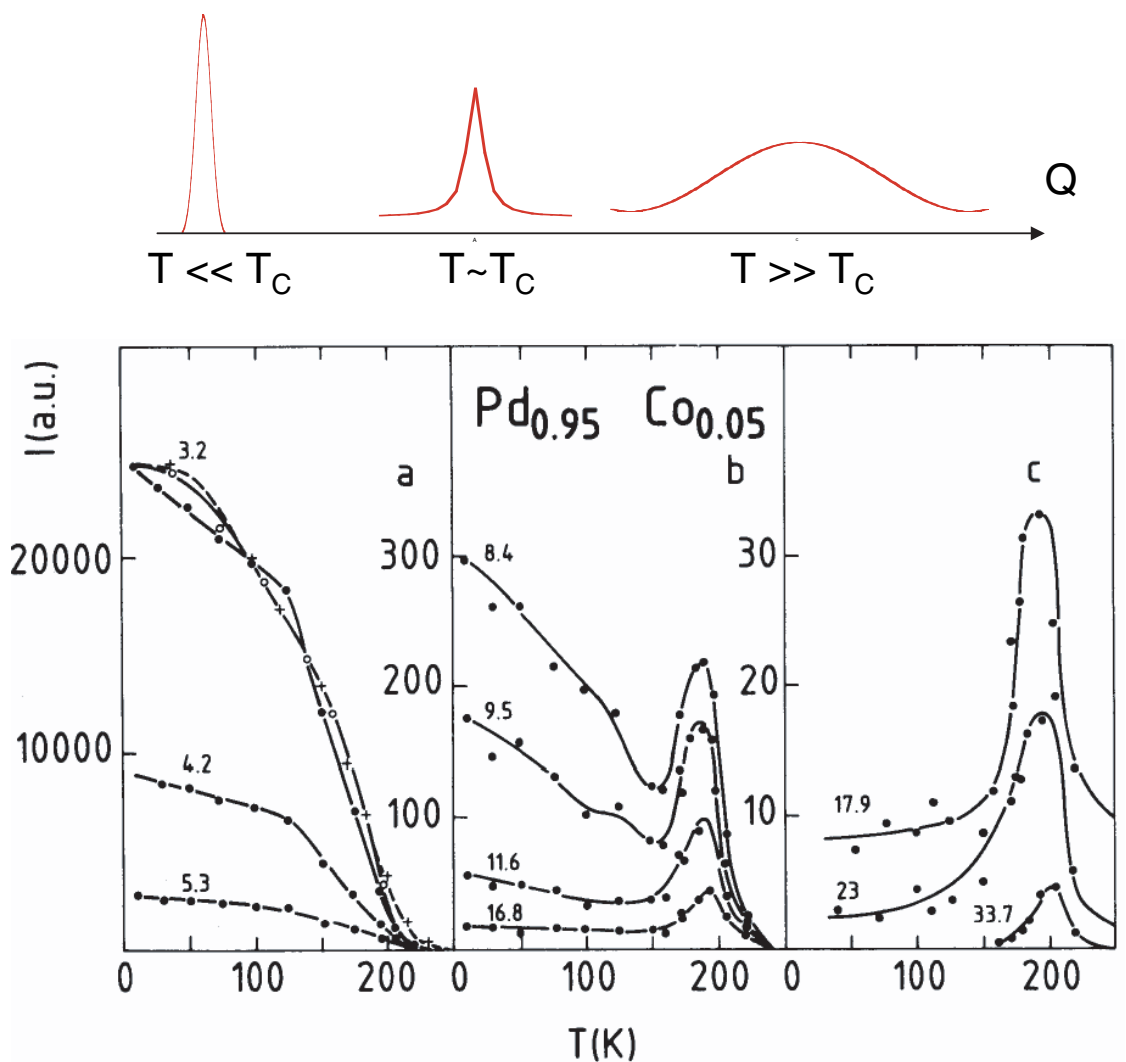

Figure 7. En haut représentation schématique de la modulation d'intensité avec le vecteur de diffusion au voisinage d'un phénomène critique. En bas, intensité diffusée aux petits angles dans l'alliage $\mathrm{Pd}_{0.95} \mathrm{Co}_{0.05}$, pour différents vecteurs de diffusion $\mathrm{Q}$, en fonction de la température ; la valeur de $\mathrm{q}$ en $\mathrm{nm}^{-1}$ est notée sur chaque courbe. D'après [26].

moment géant d'environ 10 magnétons de Bohr par atome d'impureté, qui s'étend sur une vingtaine d'Angströms [28]. Au delà d'une concentration de soluté de l'ordre du \% les nuages se recouvrent et l'alliage devient ferromagnétique, avec une température de Curie $\mathrm{T}_{\mathrm{C}}$ qui augmente d'environ $40 \mathrm{~K}$ par $\%$ de soluté. Ces composés constituent donc un bon exemple pour l'étude des phénomènes prècurseurs d'une transition critique.

Le schéma de la Fig. 7 permet de visualiser qualitativement ce que "voient" les neutrons en pareil cas. Dans la région paramagnétique ( $\left.\mathrm{T} \gg \mathrm{T}_{C}\right)$, les larges modulations diffuses liées à l'ordre magnétique local, visibles à grands vecteurs de diffusion (de l'ordre de $1 \AA^{-1}$ ) permettent de déterminer l'extension dans l'espace des moments géants. Dans la région critique $\left(T \sim \mathrm{T}_{C}\right)$, la longueur de corrélation magnétique augmente fortement : elle diverge à la transition. L'intensité diffusée aux petits angles s'exprime comme une Lorentzienne $\kappa /\left(\kappa^{2}+Q^{2}\right)$ où $\kappa$ est l'inverse de la longueur de corrélation de la perturbation magnétique. Cette intensité est mesurable aux petits angles, dans la gamme de vecteurs de diffusion entre $10^{-2}$ et $10^{-1} \AA^{-1}$. En dessous de $\mathrm{T}_{C}$, on observe la contribution des inhomogénéités de taille mésoscopique (la centaine ou le millier d'Angströms), dont la dépendance en $\mathrm{Q}$ est trés forte (typiquement $\mathrm{Q}^{-4}$ à $\mathrm{Q}^{-8}$ ), et donc dominante à trés petits vecteurs de diffusion (dans la gamme de $\mathrm{Q}$ de $10^{-3}$ à $10^{-2} \AA^{-1}$ ).

Le phénoméne critique est facilement observable en traçant l'intensité diffusée en fonction de la température pour un vecteur de diffusion donné (Fig. 7). Dans l'alliage $\mathrm{Pd}_{0.95} \mathrm{Co}_{0.05}$, l'intensité du pic 
de diffusion critique, visible à $\mathrm{T}_{C}$ (Fig. 7 à droite), est d'autant plus élevée que le vecteur de diffusion $\mathrm{Q}$ est plus petit : $\kappa$ tend vers zéro à $\mathrm{T}_{C}$, comme $\left(\left(\mathrm{T}-\mathrm{T}_{C}\right) / \mathrm{T}_{C}\right) s^{-v}$, où $v$ est l'exposant critique de la longueur de corrélation.

En dessous de $\mathrm{T}_{C}$, l'intensité liée à la diffusion critique décroît. A très petits vecteurs de diffusion (Fig. 7 à gauche), l'intensité magnétique est dominée par la contribution d'inhomogénéités (parois de Bloch, dislocations ou défauts magnétiques de taille mésoscopique), qui varie en température comme un pic de Bragg magnétique, autrement dit comme le carré de l'aimantation, paramétre d'ordre de la transition. Dans la gamme de Q intermédiaires (Fig. 7 au centre) la contribution des fluctuations critiques et celle des inhomogénéités statiques sont toutes deux observables.

Les mesures de diffusion aux petits angles permettent donc de déterminer certains paramètres de la région critique. Il importe de bien choisir les conditions expérimentales (gamme de vecteur de diffusion, gamme de température), suivant le phènoméne àètudier [24].

\section{ORDRE MAGNÉTIQUE LOCAL INDUIT PAR LA FRUSTRATION GÉOMÉTRIQUE}

Dans les composés géométriquement frustrés, la géométrie particuliére du réseau ne permet pas de statisfaire toutes les interactions magnétiques entre les atomes premiers voisins. Un cas d'école est celui de trois moments couplés antiferromagnétiquement aux sommets d'un triangle. Ces réseaux sont pour la plupart composés de triangles ou tétraèdres jointifs par les sommets, et donc peu connectés. Les réseaux les plus connus sont en dimension 2, le réseau Kagome (formé de triangles disposés en étoiles et composant le motif traditionnel des paniers japonais) et en dimension 3 le réseau pyrochlore, composé de tétraèdres (Fig. 8).

L'impossibilité de satisfaire toutes les interactions induit un paysage énergétique particulier, où beaucoup (éventuellement une infinité) de configurations ont la même énergie. En conséquence, dans des composés chimiquement ordonnés, des ordres magnétiques locaux originaux, possédant une grande dégénérescence de l'état fondamental, peuvent être stabilisés. Ces types d'ordre magnétiques ont reçu le nom de liquides de spin, glaces de spin ou verres de spin sans désordre chimique. Ces ordres
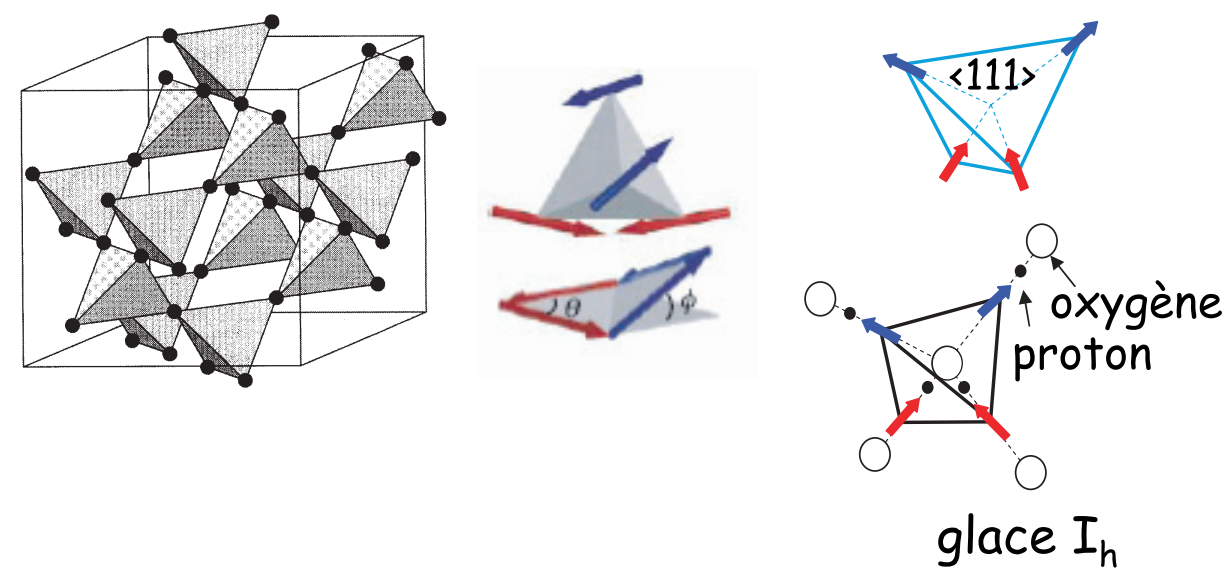

Figure 8. À gauche, le réseau pyrochlore, composé de tétraèdres connectés par les sommets. Au milieu, degrés de libertés des moments magnétiques pour les configurations qui minimisent l'énergie sur un tétraèdre dans le cas d'interactions antiferromagnétiques entre spins Heisenberg (état liquide de spin). À droite : la configuration "glace de spin", minimisant l'énergie entre moments magnétiques couplés ferromagnétiquement et contraints à s'aligner le long d'axes d'anisotropie locaux $\langle 111\rangle$. En dessous, la configuration des protons dans la glace cubique. Les flèches, analogues aux moments magnétiques, matérialisent les vecteurs joignant le centre de gravité de la liaison O-O à l'atome $\mathrm{H}$. 

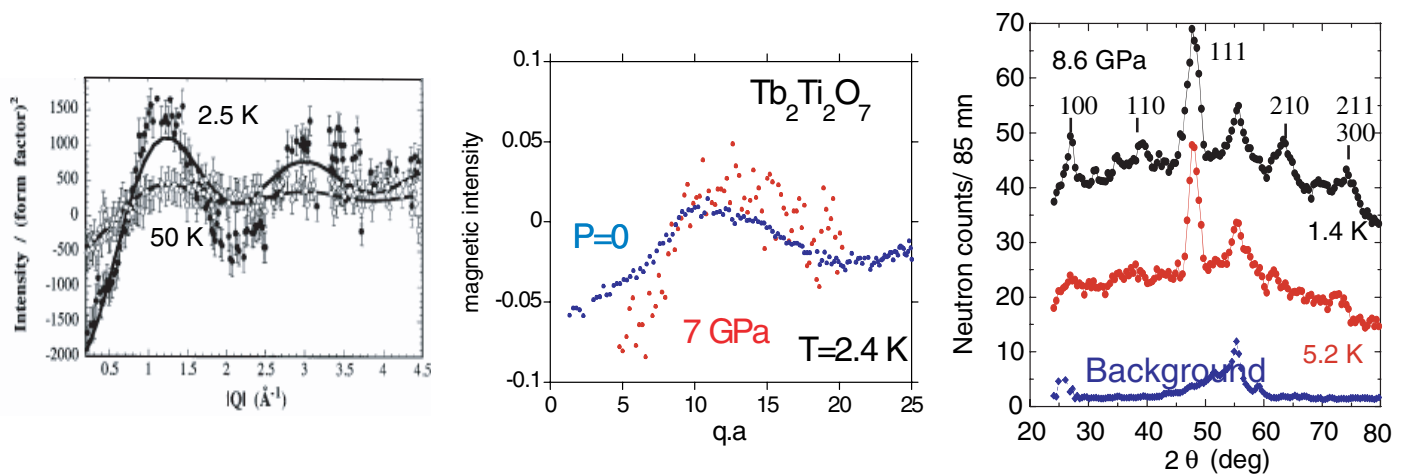

Figure 9. À gauche : spectre de diffraction magnétique de neutrons sur poudre dans $\mathrm{Tb}_{2} \mathrm{Ti}_{2} \mathrm{O}_{7}$ à $2.5 \mathrm{~K}$, montrant la présence de corrélations à courte portée (état liquide de spin). Un spectre de diffraction à $100 \mathrm{~K}$, dans la région paramagnétique où les moments sont décorrélés a été soustrait afin d'isoler la contribution magnétique. La courbe en traits pleins est un ajustement par une fonction de corrélation entre moments magnétiques premiers voisins. D'après la référence [30]. Au centre : spectre de diffraction magnétique de poudre dans la phase liquide de spin, à pression ambiante et sous pression. Les corrélations antiferromagnétiques sont renforcées sous pression. À droite : spectre de diffraction magnétique sous pression de $8.6 \mathrm{GPa}$; l'apparition d'un ordre antiferromagnétique à longue portée en dessous de $2 \mathrm{~K}$ est mise en évidence par les pics de Bragg magnétiques du réseau cubique simple. D'après la référence [31].

magnétiques originaux ont suscité beaucoup d'intérêt depuis une dizaine d'années, tant d'un point de vue expérimental que théorique.

L'état "liquide de spin" peut être stabilisê pour des interactions antiferromagnétiques entre moments isotropes, et peut se décrire trés schématiquement par la présence de corrélations de premiers voisins entre moments dont l'orientation fluctue dans l'espace et dans le temps (Fig. 8 au centre). L'état "glace de spin", stabilisé pour des interactions ferromagnétiques entre moments anisotropes (i.e. contraints à demeurer le long des axes locaux de type $\langle 111\rangle$ du tétraèdre, (Fig. 8 à droite) présente des analogies avec la glace cubique et la même entropie liée au désordre dans l'état fondamental [29]. L'état "verre de spin", contrairement aux deux précèdents, présente une transition de nature coopérative à une température bien définie, vers un état magnétique désordonné et gelé.

La complexité du paysage énergétique est telle qu'une faible perturbation peut permettre de stabiliser une nouvelle phase. L'application d'une pression permet de changer l'équilibre énergétiques entre les différentes interactions (échange direct, super-échange, interactions dipolaires, anisotropie etc.) qui dépendent de façon différentes des distances interatomiques. On peut donc observer sous pression l'apparition de phases magnétiques originales.

\subsection{Un liquide de spin s'ordonne sous pression}

Dans le pyrochlore de terbium $\mathrm{Tb}_{2} \mathrm{Ti}_{2} \mathrm{O}_{7}$, les ions $\mathrm{Tb}^{3+}$ occupent les sommets d'un réseau pyrochlore et les ions $\mathrm{Ti}^{4+}$ sont non magnétiques. Le composé présente à pression ambiante un état liquide de spin, caractérisé par des corrélations antiferromagnétiques entre moments magnétiques premiers voisins [30], fluctuant dans l'espace et dans le temps, jusqu'aux plus basses températures mesurées (environ $50 \mathrm{mK}$ ) (Fig. 5.1 gauche). Sous pression, les corrélations magnétiques sont modifiées (Fig. 5.1 centre), et un ordre antiferromagnétique à longue portée apparaît (Fig. 5.1 droite) [31]. Des mesures sur monocristaux [32] ont permis de compléter les mesures initiales sur poudres [31] et de déterminer l'origine physique de la "cristallisation" du liquide de spin, due au couplage magnéto-élastique. L'application d'une pression hydrostatique, combinée à celle d'un pression uniaxiale, renforce les interactions d'échange, mais induit surtout une légère déformation des tétraèdres, et des liaisons magnétiques non compensées. Certaines 
liaisons sont renforcées, d'autres affaiblies, suivant la direction de la contrainte et l'angle entre celle-ci et les liaisons considérées. Il est alors possible de piloter par la contrainte la température de Néel et l'amplitude du moment ordonné à longue portée.

\subsection{Une glace de spin induite par les interactions dipolaires}

Dans les pyrochlores de terres rares, la sélection des états magnétiques ordonnés à courte portée de type liquides, glaces ou verres de spin, est régie par la nature des interactions magnétiques (ferro ou antiferromagnétique) et l'anisotropie de champ cristallin. Les composés $\mathrm{Ho}_{2} \mathrm{Ti}_{2} \mathrm{O}_{7}$ et $\mathrm{Ho}_{2} \mathrm{Sn}_{2} \mathrm{O}_{7}$, dans lesquels l'anisotropie de l'Holmium est trés forte (les moments de Ho sont contraints de s'aligner sur les axes locaux de type $\langle 111\rangle$ qui joignent le centre d'un tétraèdre à ses sommets), montrent un comportement "glace de spin" [33, 34]. L'interaction effective géométriquement frustrée est de nature globalement ferromagnétique, combinant l'interaction de super-échange (antiferromagnétique entre premiers voisins) et l'interaction dipolaire, oscillante et à plus longue portée.

Les mesures de l'ordre magnétique local par diffusion de neutrons [34] sont cruciales pour établir l'origine de ce comportement. La section efficace liée à l'ordre magnétique local a été calculée pour des spins Ising en champ moyen [35] dans le cas d'interactions de proches voisins ferromagnétiques ou antiferromagnétiques, et d'interactions dipolaires. (Fig. 10 à droite). L'expérience (Fig. 10 à gauche) confirme l'influence des interactions dipolaires. La forte anisotropie de la terre rare (Ho ou Dy) est essentielle à la stabilisation de l'ordre local "glace de spin". Dans le cas de $\mathrm{Tb}_{2} \mathrm{Sn}_{2} \mathrm{O}_{7}$ où l'anisotropie du $\mathrm{Tb}$ est plus faible, la dégénérescence de l'état fondamental est partiellement levée, et la "glace de spin" s'ordonne à longue distance [36].
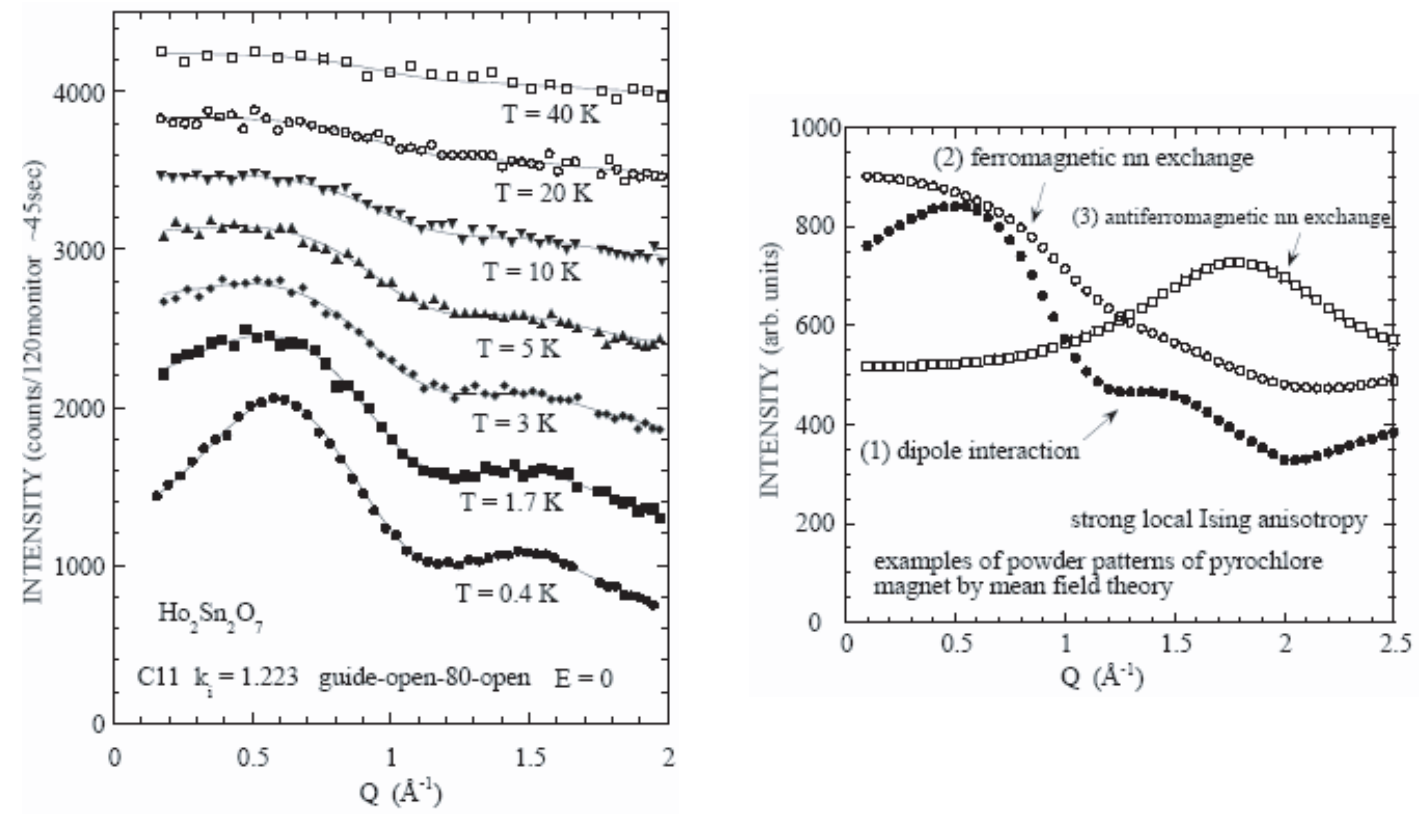

Figure 10. A gauche, la section efficace liée à l'ordre magnétique local mesurée dans la glace de $\operatorname{spin} \mathrm{Ho}_{2} \mathrm{Sn}_{2} \mathrm{O}_{7}$. A droite, le calcul de la section efficace magnétique en champ moyen sur le réseau pyrochlore dans le cas de spins Ising couplés par une interaction de proches voisins ferromagnétiques ou antiferromagnétiques, ou par une interaction dipolaire. La comparaison entre expérience et calcul permet de visualiser l'influence des interactions dipolaires sur la stabilisation de l'ordre local "glace de spin". D'après la référence [35]. 


\subsection{Transition ferromagnétique-verre de spin induite sous pression}

Dans le molybdate de terbium, $\mathrm{Tb}_{2} \mathrm{Mo}_{2} \mathrm{O}_{7}$ les ions $\mathrm{Tb}^{3+}$ et $\mathrm{Mo}^{4+}$ sont tous deux magnétiques et situés aux sommets de deux réseaux de tétraèdres jointifs par les sommets, décalés l'un de l'autre par un vecteur [1/21/21/2]. Le magnétisme localisé de l'ion de terre rare $\mathrm{Tb}^{3+}$, qui possède une anisotropie Ising locale (type "glace de spin") interagit avec celui partiellement itinérant et isotrope de l'ion de transition $\mathrm{Mo}^{4+}$. Le changement de la structure de bande lié à la variation des distances interatomiques donne lieu à toute une physique intéressante [37], pouvant conduire à des propriétés de conduction originales, comme l'effet Hall anormal géant [38]. Bien qu'ordonné chimiquement, le composé $\mathrm{Tb}_{2} \mathrm{Mo}_{2} \mathrm{O}_{7}$ présente une transition coopérative vers un état "verre de spin" à $\mathrm{T}_{\mathrm{G}}=25 \mathrm{~K}$ [39], sans doute induite par un couplage magnéto-élastique avec les déformations spontanées du réseau.

Les corrélations à courte portée de l'état fondamental verre de spin de $\mathrm{Tb}_{2} \mathrm{Mo}_{2} \mathrm{O}_{7}$ sont clairement visibles sur le spectre magnétique de la Fig. 11 (en haut à gauche). En dilatant le réseau $\mathrm{Tb}^{3+}$ par $^{2}$ substitution partielle d'ion $\mathrm{La}^{3+}$, non magnétique mais plus gros, il est alors possible de modifier la structure de bande et d'induire un ordre ferromagnétique non colinéaire à longue portée (Fig. 11 à gauche) [40]. Dans l'échantillon substitué $\left(\mathrm{Tb}_{1-x} \mathrm{La}_{x}\right)_{2} \mathrm{Mo}_{2} \mathrm{O}_{7}, \mathrm{x}=0.2$, l'état verre de spin réapparaît sous pression appliquée (Fig. 11 à droite). L'évolution des corrélations de spin est clairement visible à toutes les étapes de cette transformation : les modulations sinusoidales liées aux corrélations de premiers voisins (Fig. 11 en haut) laissent la place à des corrélations mésoscopiques de nature Lorentzienne, à l'échelle de la vingtaine d'Angströms, (Fig. 11 au centre), puis à des pics de Bragg (Fig. 11 en bas). Ces spectres de diffraction ont été simulés quantitativement par des modèles appropriés, permettant d'obtenir

\section{Dilatation du réseau}

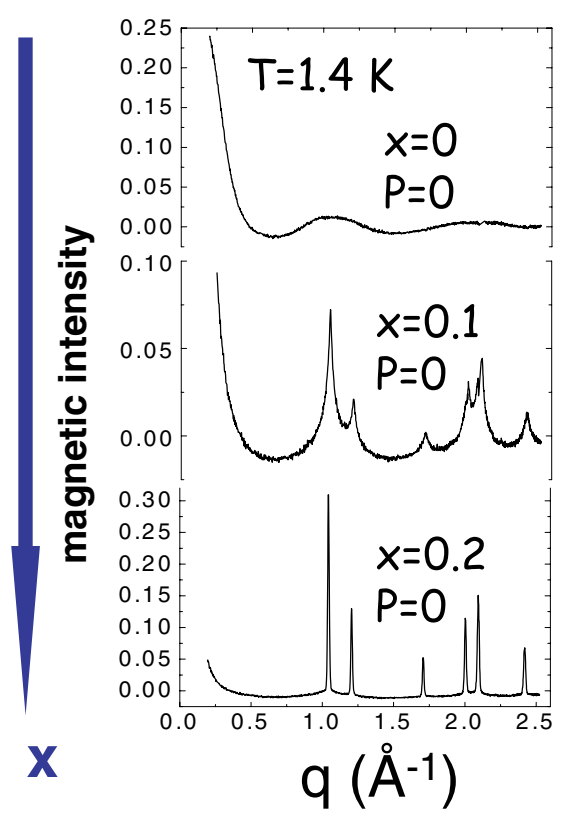

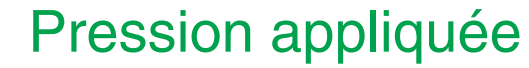

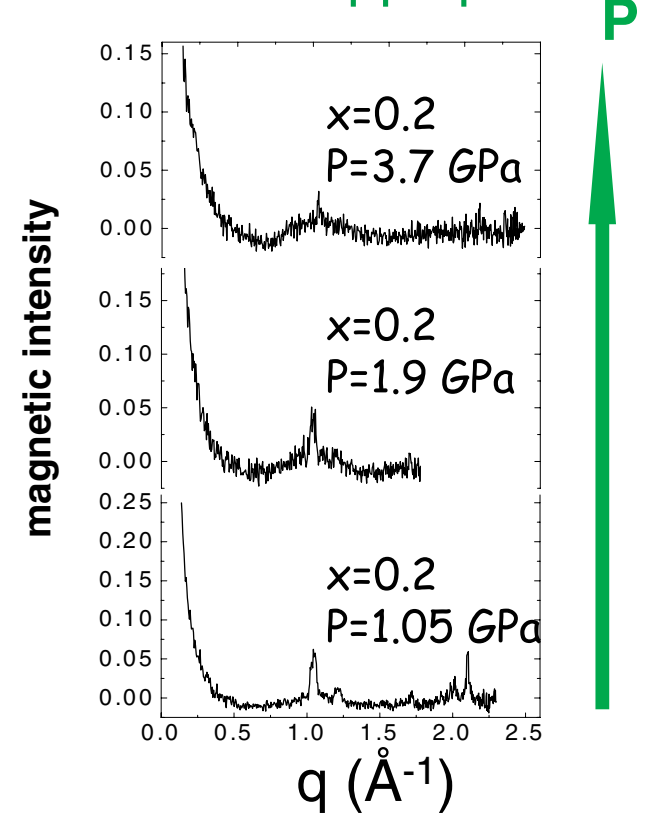

Figure 11. Spectre de diffraction magnétique à $1.4 \mathrm{~K}$ dans $\left(\mathrm{Tb}_{1-\mathrm{x}} \mathrm{La}_{\mathrm{x}}\right)_{2} \mathrm{Mo}_{2} \mathrm{O}_{7}$. À gauche, dans le composé verre de spin $\mathrm{Tb}_{2} \mathrm{Mo}_{2} \mathrm{O}_{7}$, un ordre magnétique à longue portée est induit par la substitution partielle des ions $\mathrm{Tb}^{3+}$ par des ions $\mathrm{La}^{3+}$ plus gros qui dilatent le réseau. À droite, dans le composé substitué $\left(\mathrm{Tb}_{1-\mathrm{x}} \mathrm{La}_{\mathrm{x}}\right)_{2} \mathrm{Mo}_{2} \mathrm{O}_{7}, \mathrm{x}=0.2$, l'application d'une pression permet de recouvrer l'état verre de spin, où seules persistent des corrélations à courte portée. D'après la référence [40]. 
toutes les caractéristiques microscopiques (moments ordonnés, paramètres d'ordre local, longueurs de corrélations) des différentes étapes de la transformation de l'état fondamental magnétique sous pression chimique ou appliquée [41].

\section{CONCLUSION}

Ce chapitre donne seulement un petit aperçu de la richesse et de la variété des phénomènes physiques que la diffraction de neutrons permet d'appréhender dans le domaine de l'ordre local. On s'est limité ici à quelques cas simples et illustratifs. Il faut garder présent à l'esprit qu'il existe des modélisations plus complexes et quantitatives. En diffraction de neutrons, l'étude du fond diffus lié à l'ordre local est souvent plus longue (il faut le mesurer avec une bonne statistique dans un grand domaine de l'espace réciproque) et plus difficile (il faut l'évaluer quantitativement, puis trouver des modèles adaptés pour le simuler) que celle de l'ordre à longue portée. Elle peut être associée à celle des fluctuations par diffusion inélastique de neutrons : l'ordre magnétique local en phase paramagnétique fluctue à des échelles de temps accessibles aux neutrons. Ce peut être aussi le cas de l'ordre chimique local, pour des déplacements atomiques d'atomes légers, comme l'hydrogéne. Les progrés d'analyse récents concernent par exemple la modélisation du fond diffus par des techniques de Monte-Carlo inverse, l'analyse de la section efficace totale comme dans les liquides ou amorphes, par transformée de Fourier de la fonction de corrélation de paires, qui permet de s'abstraire de la présence d'un réseau cristallin, si nécessaire. L'étude des cas simples que sont les alliages cristallins binaires et la définition des concepts de base associés à l'ordre local, peut permettre d'aborder plus facilement ces domaines en pleine expansion.

\section{Remerciements}

Isabelle Mirebeau remercie Brigitte Beuneu et Véronique Pierron-Bohnes pour d'utiles discussions et une lecture critique du manuscript.

\section{Références}

[1] Cowley J. M., Phys. Rev. 77, 669 (1950); Phys. Rev. 138, A1384 (1965).

[2] Warren B. E., X ray diffraction, Addison-Wesley Publishing Comp., 1968.

[3] Clapp P. C. and Moss S.C., Phys. Rev. 142, 418 (1966).

[4] Mehaddene T., Sanchez J. M., Caudron R., Zemirli M. and Pierron-Bohnes V., Eur. Phys. B 41, 207 (2004).

[5] Storms E. K., The Refractory Carbides (Academic Press, New York, 1967).

[6] Borie B. and Sparks C. J., Acta. Cryst. A 27, 198 (1971).

[7] Priem T., Beuneu B., de Novion Ch., Chevrier J., Livet F., Finel A. and Lefebvre S., Physica B 156-157, 47 (1989); Thierry Priem, Thése d'état, Université Paris-Sud (1988).

[8] Caudron R. et al., J. Phys. I France, 2, 1145 (1992).

[9] Balanzat E. and Hillairet J., J. Phys. F., Met. Phys., 11, 1977 (1981).

[10] Pierron-Bohnes V., Mirebeau I., Balanzat E. and Cadeville M. C., J. Phys. F., Met. Phys., 14, 197 (1984).

[11] Rossiter P. L., The Electrical Resistivity of Metals and Alloys (Cambridge University Press, 1987).

[12] Mirebeau I., Hennion M. and Parette G., Phys. Rev. Lett. 53, 687 (1984).

[13] Hennion M., J. Phys. F. 13, 2351 (1983).

[14] Caro A., Crowson D. A. and Caro M., Phys. Rev. Lett. 95, 75702 (2005). 
[15] Wallenius J., Olsson P., Lagerstedt C., Sandberg N., Charakova R. and Pontikis V., Phys. Rev. B 69, 94103 (2004); Olsson P., Wallenius J., Domain C., Nordlund K. and Malerba L., Phys. Rev. B 72, 214119 (2005).

[16] Shull C. G. and Wilkinson M. K., Phys. Rev. 97, 304 (1955).

[17] Marshall W., J. Phys. C, (Proc. Phys. Soc.), Vol. 1, 88 (1968); Marshall W. and Lovesey S. W., Theory of Thermal Neutron Scattering (Oxford, Clarendon Press, 1971).

[18] Low G. G. and Collins M. F., J. Appl. Phys. 34, 1195 (1963).

[19] Aldred A. T., Rainford B. D., Kouvel J. S. and Hicks T. J., Phys. Rev. B 14, 228 (1976).

[20] Drittler B., Stefanou N., Blugel S., Zeller R. and Dederichs P., Phys. Rev. B 40, 8203 (1989).

[21] Parette G., résultats non publiés.

[22] Bertaut E. F. and Burlet P., Solid State. Com. 5, 279 (1967); Wiedenmann A., Burlet P., Scheuer H. and Convert P., Solid State. Com. 38, 129 (1981).

[23] Greedan J. E., Reimers J. N., Stager C. V. and Penny S. L., Phys. Rev. B 43, 5682 (1991).

[24] voir la revue sur la Diffusion de Neutrons aux Petits Angles parue dans les cours de l'Ecole Thématique des JDN7 (1998) : J. de Phys. IV 9, 1 (1999).

[25] Jaquet V., Thése de doctorat de l'Ecole Polytechnique (2000).

[26] Mirebeau I., Hennion M., Hippert F. and Campbell I. A., J. Mag. Mag. Mat. 54-57, 997 (1986).

[27] Bellouard C., Mirebeau I. and Hennion M., Phys. Rev. B 53, 5570 (1996).

[28] Crangle J. and Scott W. R. J. Appl. Phys. 36, 921 (1965); Nieuwenhuys G. J., Advan. Phys. 24, 515 (1975).

[29] Ramirez A. P., Hayashi A., Cava R. J., Siddharthan R. and Shastry B. S., Nature 399, 333 (1999).

[30] Gardner J. S. et al., Phys. Rev. Lett. 82, 1012 (1999).

[31] Mirebeau I., Goncharenko I., Cadavez-Peres P., Bramwell S. T., Gingras M. J. P. and Gardner J. S., Nature 420, 54 (2002).

[32] Mirebeau I., Goncharenko I., Dhalenne G. and Recolevschi A., Phys. Rev. Lett. 93, 187204 (2004).

[33] Bramwell S. T. and Gingras M. J. P. , Science 294, 1495 (2001).

[34] Harris M. J., Bramwell S. T., McMorrow D. F., Zeiske T. and Godfrey K. W., Phys. Rev. Lett. 79, 2554 (1997).

[35] Kadowaki H., Ishii Y., Matsuhira K. and Inatsu Y., Phys. Rev. B 65, 144421 (2002).

[36] Mirebeau I. et al., Phys. Rev. Lett. 94, 246402 (2005).

[37] Solovyev I. V., Phys. Rev. B 67, 174406 (2003).

[38] Taguchi Y., Oohara Y., Yoshisawa H., Nagaosa N. and Tokura Y., Science 291, 2573 (2001).

[39] Gaulin B. D., Reimers J. N., Mason T. E.,. Greedan J. E. and Tun Z., Phys. Rev. Lett. 69, 3244 (1992).

[40] Apetrei A., Mirebeau I., Goncharenko I., Andreica D. and Bonville P., Phys. Rev. Lett. 97, 206401 (2006).

[41] Apetrei A., Thése de doctorat, Université d'Orsay (2007). 